\title{
FICORREMEDIACIÓN DE AGUAS RESIDUALES URBANAS DE PEQUEÑOS MUNICIPIOS CON MICROALGAS
}

\section{PHYTOREMEDIATION OF URBAN WASTEWATER OF SMALL MUNICIPALITIES WITH MICROALGAE}

MSc. Manuel Domínguez Gómez

Máster de Gestión Integral del Agua (España).

Labora en el Instituto Universitario de Investigaciones Marinas (INMAR) en la Universidad de Cádiz, España. manuel.dominguez@uca.es

Dr. C. Jesús Ruiz González Doctor en Ciencias (España). Profesor interino de la Universidad de Cádiz, CEO de la spinoff ALGADES, España. jesus.ruiz@algades.com

Dra. C. Carmen Garrido Pérez Doctora en Ingeniería Química (España). Profesora Titular de Tecnologías del Medio Ambiente de la Universidad de Cádiz, España. carmen.garrido@uca.es

\section{Dr. C. José Antonio Perales Vargas-Machuca}

Doctor en Ingeniería Química (España).

Catedrático de Tecnologías del Medio Ambiente de la Universidad de Cádiz, España. joseantonio.perales@uca.es

\section{ARTÍCULO DE REVISIÓN}

Recibido: 22 de mayo de 2020.

Aceptado: 4 de junio de 2020. 


\section{RESUMEN}

La eliminación de nutrientes ( $N$ y $\mathrm{P}$ ) en estaciones depuradoras de aguas residuales de pequeños municipios < 2000 habitantes, es actualmente un reto pues la tecnología disponible actualmente es de carácter intensivo, con costes de inversión y operación inasumible en aglomeraciones urbanas de pequeño tamaño. La ficorremediación de aguas residuales utilizando consorcios microalgas-bacteria, es una de las tecnologías más prometedoras para el tratamiento y eliminación de materia orgánica y nutrientes, de las aguas residuales de tipo urbana de los pequeños núcleos de población. En este artículo, se analiza esta tecnología incidiendo en sus ventajas, así como los principales hándicaps sobre los que poner más empeño en investigación para conseguir que la biotecnología de microalgas en el campo de la depuración de aguas en pequeños municipios sea una alternativa económicamente viable.

Palabras clave: aguas residuales urbanas, ficorremediación, fotorreactores, microalgas

\section{ABSTRACT}

The removal of nutrients ( $N$ and $P$ ) in wastewater treatment plants of small municipalities $<2000$ inhabitants, is currently a challenge since the technology currently available is intensive, with investment and operation costs unaffordable in small urban agglomerations. The wastewater phycoremediation using microalgae-bacteria consortia is one of the most promising technologies for the treatment and removal of organic matter and nutrients from urban wastewater from small populations. In this article, this technology is analysed, emphasizing its advantages as well as the main handicaps on which to put more research effort to make microalgae biotechnology in the field of wastewater treatment in small municipalities an economically viable alternative. 
Keywords: urban wastewater, phytoremediation, photoreactors, microalgae

\section{INTRODUCCIÓN}

La principal fuente de contaminación por nutrientes al medio hídrico son las escorrentías de los suelos agrícolas que han sido sobrefertilizados. Este tipo de contaminación difusa solo se puede controlar aplicando correctamente los fertilizantes, y solo en la cantidad necesaria. En cuanto a las fuentes puntuales de contaminación por nutrientes, la principal entrada al medio proviene de aguas residuales urbanas e industriales. Estos tipos de contaminación son controlables antes de llegar al medio receptor, pero generalmente los tratamientos que se aplican son inadecuados o insuficientes para reducir convenientemente la carga de vertido de nutrientes a los medios acuáticos (Carey y Migliaccio, 2009). Así, se estima que los vertidos anuales globales de nitrógeno y fósforo procedentes de aguas residuales supondrán para 2050 entre $12,0-15,5 \cdot 10^{3}$ toneladas de nitrógeno $(\mathrm{N})$ y $2,4-3 \cdot 10^{3}$ toneladas de fósforo $(\mathrm{P})$, observándose un incremento de alrededor de 3 veces el valor que había en el año 2000 (Van Drecht et al., 2009).

En Europa, conforme a la Directiva 91/271/CEE sobre el tratamiento de las aguas residuales urbanas, las concentraciones de nitrógeno y fósforo en los efluentes de estaciones depuradoras de aguas residuales (EDAR) de carga contaminante superior a 10.000 h-e (habitantes equivalentes, tomando como referencia la carga contaminante de un habitante como $60 \mathrm{~g} / \mathrm{d}$ de $\mathrm{DBO}_{5}$ ), y que viertan a zonas catalogadas como "sensibles" a sufrir procesos de eutrofización, deben ser controladas mediante la aplicación de "tratamientos rigurosos" hasta alcanzar concentraciones o rendimientos de eliminación aceptables. Asimismo, esta normativa establece que, para poblaciones más pequeñas, 0 pequeñas aglomeraciones ( $<2.000 \mathrm{~h}-\mathrm{e})$, donde la aplicabilidad de tecnologías intensivas de tratamiento no es viable, las aguas residuales han de someterse a un "tratamiento adecuado", que garantice la calidad y los usos de las aguas receptoras de estos vertidos.

Los vertidos de aguas residuales procedentes de pequeñas aglomeraciones urbanas, si bien globalmente podrían suponer una fuente menor de contaminación por la carga contaminante que generan, a nivel local puede suponer un serio problema en la calidad de sus aguas receptoras. Para estos municipios, la depuración de las aguas residuales solo resulta económicamente viable mediante el uso de las tecnologías extensivas (Tabla 1), 
con bajos requerimientos energéticos y escasa mano de obra, ya que, a diferencia de las EDAR de grandes núcleos urbanos, no se pueden beneficiar de la economía de escala. En la Tabla 1 se recogen algunas de las tecnologías de depuración más empleadas en núcleos urbanos de menos de 10.000 h-e.

Tabla 1. Rendimientos de eliminación de contaminantes y área que ocupa distintas tecnologías de depuración.

\begin{tabular}{|c|c|c|c|c|c|c|c|}
\hline \multicolumn{2}{|c|}{ Tecnología } & SS (\%) & $\mathrm{DBO}_{5}(\%)$ & DQO (\%) & $\mathrm{N}(\%)$ & $\mathrm{P}(\%)$ & $\mathrm{m}^{2} / \mathrm{hab}$ \\
\hline \multirow{3}{*}{$\begin{array}{c}\text { Aplicación al } \\
\text { Terreno }\end{array}$} & Filtro Verde & 95-99 & $90-95$ & $90-94$ & $90-95$ & $85-90$ & $12-110$ \\
\hline & Infiltración rápida & $90-95$ & $90-95$ & $70-80$ & $30-95$ & $25-40$ & $2-22$ \\
\hline & $\begin{array}{l}\text { Escorrentía } \\
\text { superficial }\end{array}$ & $70-80$ & 90-95 & $60-70$ & $45-50$ & $20-30$ & $5-15$ \\
\hline \multirow{3}{*}{ Lagunajes } & Anaerobia & $50-65$ & $40-50$ & $40-50$ & $10-5$ & $0-5$ & $1-3$ \\
\hline & Facultativa & $0-70$ & $60-80$ & $55-75$ & $30-60$ & $0-30$ & $2-20$ \\
\hline & Maduración & $40-80$ & $75-85$ & $70-80$ & $35-80$ & $1-60$ & $1-3^{*}$ \\
\hline \multicolumn{2}{|c|}{ Humedal artificial } & $85-95$ & $80-90$ & $75-85$ & $20-40$ & $15-30$ & $3-8$ \\
\hline \multirow{3}{*}{$\begin{array}{c}\text { Procesos } \\
\text { biológicos de } \\
\text { cultivo fijo }\end{array}$} & Lechos turba & $80-90$ & $75-85$ & $70-80$ & $40-50$ & $20-30$ & $3-5$ \\
\hline & Biodiscos & $80-85$ & $80-85$ & $80-85$ & $8-15$ & $10-20$ & $0,5-0,7$ \\
\hline & Lecho bacteriano & $85-95$ & $85-95$ & $80-90$ & $20-35$ & $10-35$ & $0,5-0,7$ \\
\hline $\begin{array}{l}\text { Procesos } \\
\text { biológicos de } \\
\text { cultivo en } \\
\text { suspensión }\end{array}$ & Lodos activos & $80-90$ & 80-95 & $80-90$ & $10-30$ & $8-25$ & $0,2-1^{* *}$ \\
\hline
\end{tabular}

**Considerando 0,2 para lodos activos de media carga y 1 para aireación prolongada, teniendo en cuenta que el THR del primero es 5 veces más pequeño y suponiendo la misma profundidad. SS: sólidos en suspensión; $\mathrm{DBO}_{5}$ : demanda biológica de oxígeno medida a los 5 días; DQO: demanda química de oxígeno

Fuentes: Collado Lara, 1992; Tchobanoglous et al., 1995; Salas Rodríguez y Agencia Andaluza del Agua, 2007.

Las tecnologías más usuales para la eliminación de nitrógeno son de carácter intensivo y se basan en procesos de nitrificación aerobia seguido de una desnitrificación en condiciones anóxicas. Las tecnologías para la eliminación de fósforo son también intensivas 
y se basan en la precipitación química de fósforo mediante la adición de sales metálicas, o su eliminación biológica conjunta con el nitrógeno (p.ej. procesos Bardenpho, UCT, A²O).

Estos procesos presentan unos costes de operación elevados en comparación con sus homólogos para la eliminación de materia orgánica y sólidos en suspensión (tratamientos secundarios). Así, mientras que el coste de la eliminación de materia orgánica suele rondar entre $0,5-1 € / \mathrm{kg}$, los tratamientos de eliminación de nitrógeno y de fósforo rondan entre 5$8 € / \mathrm{kg}$ para el nitrógeno y 13-20 €/Kg para el fósforo (Bode y Grunebaum, 2000).

Para aquellas situaciones en las que sea preciso eliminar nitrógeno y fósforo y la única opción económicamente sostenible sea tecnologías de tipo extensiva, los rendimientos de eliminación alcanzables no son suficientemente altos para alcanzar una buena calidad de efluente (ver Tabla 1). A diferencia de los grandes núcleos de población, actualmente no existen tecnologías robustas y suficientemente desarrolladas para la eliminación de nutrientes para pequeñas poblaciones, esto es, con unos costes y sencillez de operación acordes a los requerimientos de estos entornos.

Como se ha comentado, la limitación de la concentración de nutrientes en vertidos de aguas residuales se prevé que se extienda a todo tipo de municipios, por ello y dada la limitación existente en poblaciones de pequeño tamaño, resulta indispensable el desarrollo de tecnologías eficaces, aplicables y económicamente sostenibles. En este escenario es donde la ficorremediación de aguas residuales mediante el uso de microalgas se posiciona como una posible solución tecnológica, siendo una opción dotada de una gran sencillez de operación repercutiendo en una menor necesidad de mantenimiento y, por tanto, con costes de personal reducidos.

\section{REVISIÓN TEÓRICA}

\subsection{Ficorremediación}

El aprovechamiento de organismos fotosintéticos para la depuración de aguas residuales es una práctica común en sistemas de tratamiento extensivos como son las lagunas facultativas o los humedales artificiales. Sin embargo, el uso específico de la biotecnología de microalgas para el tratamiento de aguas residuales no se empezó a proponer hasta la década de los años cincuenta del pasado siglo por Oswald et al. (1957). La ficorremediación 
mediante microalgas aprovecha la cinética de consumo de nutrientes de estos microorganismos, con la eliminación de otros contaminantes como fármacos (Y. Wang et al., 2017; Villar-Navarro et al., 2018), metales pesados (Wilde y Benemann, 1993; Yu y Wang, 2004), e incluso de forma general, se consigue una mayor detoxificación del agua residual (Díaz-Garduño et al., 2017). Asimismo, la ficorremediación permite promover en un mismo fotobiorreactor, el crecimiento conjunto de bacterias y microalgas estableciéndose una sinergia entre ambas poblaciones. De este modo y sin necesidad de consumir energía en aireación, es posible reducir la carga contaminante en materia orgánica y nutrientes simultáneamente (Muñoz y Guieysse, 2006).

Desde el punto de vista de la calidad del efluente, los beneficios de los procesos de ficorremediación con microalgas no solo se traducen en la generación de un efluente con bajas concentraciones de $\mathrm{N}$ y $\mathrm{P}$ y otros microcontaminantes, sino que además dicho efluente presentará unas concentraciones elevadas de oxígeno, hasta un 300\% de saturación en horas centrales del día (Craggs et al. 1996; Park y Craggs, 2011), lo que es altamente beneficioso para el medio receptor.

Frente a los tratamientos convencionales presenta ventajas económicas y de operación: la tecnología es mucho menos compleja cuando se utilizan reactores tipo carrusel, raceway o HRAP (high rate algal or algae ponds); la inversión requerida para la instalación de un sistema basado en microalgas en comparación con un sistema de fangos activos es menos de la mitad; y los costes de operación inferiores al 20\% (Craggs et al., 2011). Estos últimos son reducidos debido a que no es necesario la adición de reactivos (Posadas et al., 2015; Duarte-Santos et al., 2016; Arbib et al., 2017), y los requerimientos energéticos son menores al no precisar aireación ya que es suficiente una agitación suave (mediante palas) para que el medio sea homogéneo y las algas no sedimenten.

Un proceso de tratamiento de aguas residuales mediante biotecnología de microalgas cuenta con dos elementos principales: (1) el fotobiorreactor; y (2) la unidad de separación biomasa-efluente denominado también proceso de cosechado (Figura 1). 


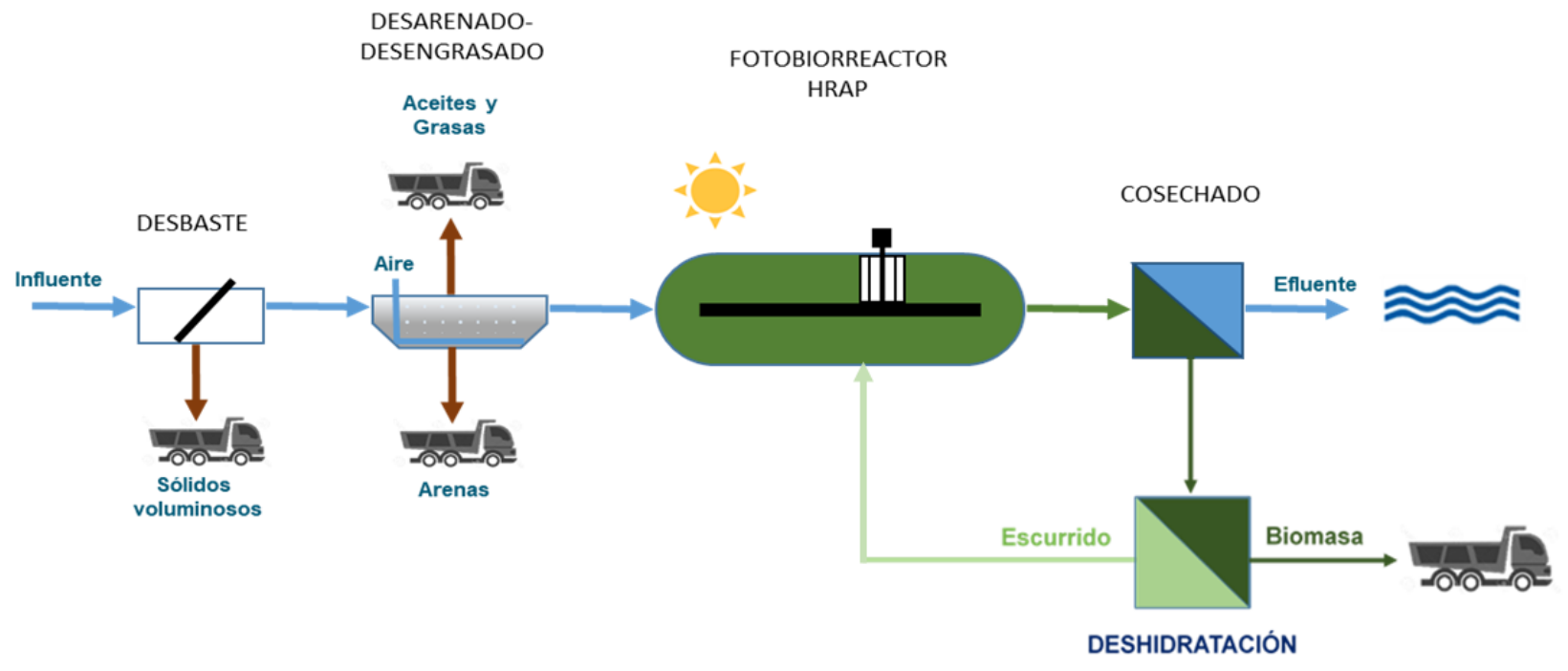

Figura 1. Diagrama de flujo general de un proceso de tratamiento de aguas residuales por ficorremediación.

\section{Fotobiorreactores}

Independientemente del tipo de fotobiorreactor y la finalidad con el que este haya sido diseñado, los criterios de diseño son los mismos tanto para sistemas de producción de microalgas para obtener productos de alto valor (Muñoz y Guieysse, 2006), como para los fotobiorreactores empleados en la depuración de aguas residuales. Estos serían:

- Una elevada relación superficie/volumen, para maximizar la disponibilidad de luz.

- Una adecuada mezcla para mantener el medio homogéneo y evitar sedimentación de las microalgas.

- Una adecuada aireación para evitar un exceso de oxígeno en el fotobiorreactor (>300\%) que generarían fenómenos de estrés oxidativo.

El funcionamiento del fotobiorreactor vendrá condicionado en gran parte por la localización geográfica, ya que condicionará los parámetros climáticos de temperatura e irradiación solar principalmente.

Generalmente, los fotobiorreactores se clasifican en dos tipos, abiertos y cerrados, según exista o no contacto directo con la atmósfera. En el campo del tratamiento de aguas 
residuales en zonas de climas templados se emplean fotobiorreactores abiertos, tanto por cuestiones técnicas asociadas al incremento de temperatura del reactor y a la generación de fouling (Arbib et al., 2013), como por cuestiones económicas relacionadas con los costes de inversión de los reactores cerrados, estas son, entre 13 y $36 € / \mathrm{m}^{2}$ de coste de construcción de los HRAP (Norsker et al., 2011) frente a un coste medio de $100 € / \mathrm{m}^{2}$ para la construcción de reactores tubulares (Janssen et al., 2003).

Dentro de los sistemas abiertos podemos distinguir entre cultivos inmovilizados y en suspensión.

\subsection{Fotobiorreactores tipo biofilm}

Los cultivos inmovilizados, fotobiorreactores tipo biofilm, destacan por tener los microorganismos adheridos en una matriz polimérica, de forma que se simplifica bastante el cosechado de la biomasa y supone un considerable ahorro de operación.

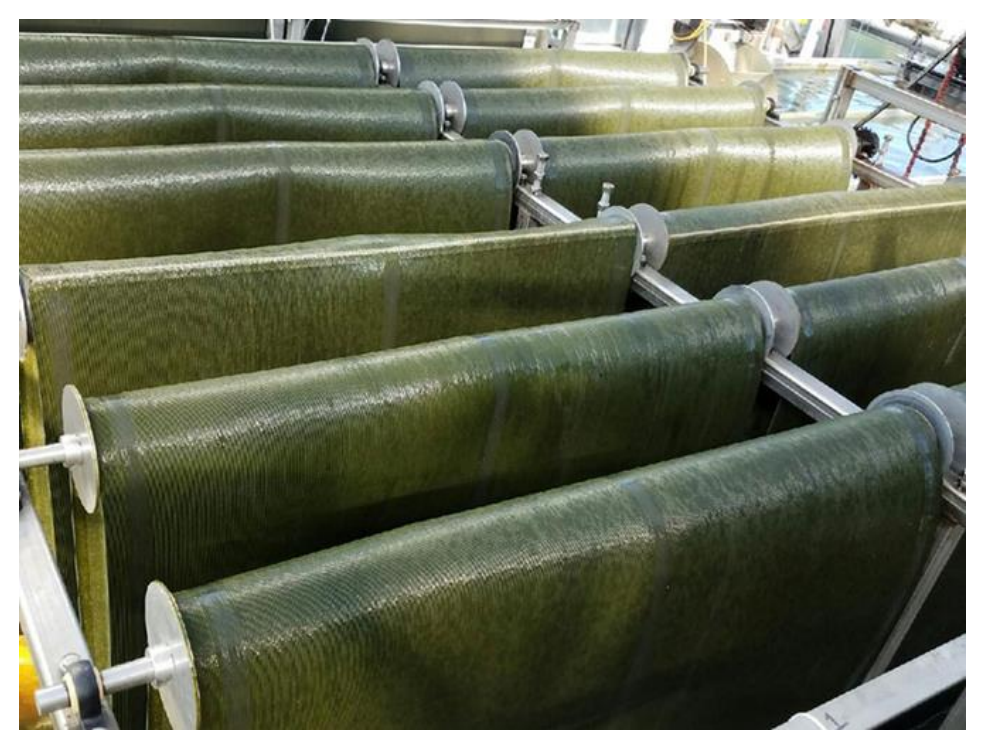

Figura 2. Fotobiorreactor de cultivo inmovilizado de la empresa Gross-Wen Technology.

Los trabajos que existen a esta escala no pasan de nivel de laboratorio (Posadas et al., 2013; Bilad et al., 2014) o de planta piloto (Christenson y Sims, 2012) y en la mayoría de éstos se trabaja tanto en mejorar las técnicas de inmovilizado (Johnson y Wen, 2010; Lee et al., 2014), como en el diseño de fotobiorreactores más eficaces (Johnson y Wen, 2010; Ozkan et al., 2012; Posadas et al., 2013). Actualmente, el reactor de biofilm rotativo es 
el que mayor eficiencia presenta en cuanto a la eliminación de nutrientes, entre 2,1 y 14,1 $\mathrm{g} / \mathrm{m}^{2}$.día de fósforo y nitrógeno, respectivamente (Christenson y Sims, 2012), y la inmovilización más efectiva se consigue con acero inoxidable, nylon y fibras naturales (Hoh et al., 2016).

Estos sistemas han demostrado eficiencias de eliminación de carbono, nitrógeno y fósforo de $91 \%, 70 \%$ y $85 \%$ respectivamente, en biorreactores inmovilizados con 10 días de tiempo hidráulico de residencia (Posadas et al., 2013). Sin embargo, a pesar de que esta estrategia muestra ventajas a la hora de obtener un efluente libre de nutrientes y biomasa, los efectos de la inmovilización sobre las células suelen ser negativos ya que el acceso limitado a la luz y al medio reduce su tasa de crecimiento y la productividad de biomasa. Se trata pues de una tecnología que aún no es aplicable a gran escala ya que la cinética de consumo de nutrientes es muy lenta y se precisa mejorar la velocidad de crecimiento de las microalgas, el transporte de materia, las interacciones algas-bacterias y ver su efectividad más allá de escala piloto (Kesaano y Sims, 2014).

En cuanto a los fotobiorreactores de cultivo en suspensión podríamos destacar dos tipos: (1) Los fotobiorreactores tipo raceway conocidos también como High Rate Algal Ponds (HRAP), y (2) los reactores tipo capa fina o thin-layer.

\subsection{Fotobiorreactores thin-layer}

Los fotobiorreactores thin-layer consisten en una superficie inclinada (1-2\%) por la que discurre el medio de cultivo junto a las microalgas, hasta un depósito inferior en el que se vuelve a bombear hasta la parte más alta. El caudal de recirculación y la pendiente determinan el espesor de la lámina de agua (trayectoria de luz). Normalmente se trabaja con profundidades de entre 0,5 y $5 \mathrm{~cm}$ por lo que su relación superficie/volumen es muy grande en comparación con el resto de sistemas (entre 200 y $20 \mathrm{~m}^{-1}$ ).

La gran exposición a la luz, el bajo consumo energético y concentraciones de biomasa de hasta $15 \mathrm{~g} / \mathrm{L}$ (Doucha y Lívanský, 1995) son algunas de las ventajas que presenta el uso de este tipo de biorreactor frente a otros. Sin embargo, la excesiva superficie que se necesita para su implantación reduce su aplicabilidad en el campo del tratamiento de aguas residuales urbanas. No obstante, varios autores han estudiado su uso para corrientes de 
aguas residuales de bajo caudal y elevada carga contaminante como es el caso de aguas residuales procedentes del sector agroalimentario/ganadero (Wang et al., 2010), o para el centrado de la deshidratación de lodos digeridos anaeróbicamente (Morales-Amaral et al., 2015) donde esta tecnología sí que presentaría un elevado potencial.

\subsection{Fotobiorreactores raceway o High Rate Algal Pond (HRAP)}

Los fotobiorreactores que más se usan a escala industrial son los sistemas tipo raceway o High Rate Algal Pond (HRAP).

Los sistemas tipo raceway consisten en un estanque poco profundo formado por dos canales separados por un tabique central. El medio circula gracias a la agitación que generan una serie de palas que giran sobre un eje horizontal. Este diseño básico ya fue sugerido hace 50 años (Oswald y Golueke, 1960), sin embargo, existen otras variantes sobre la misma idea cuyas modificaciones van enfocadas a mejorar el proceso.

La lámina de agua circulante suele tener una profundidad de entre 10 y $40 \mathrm{~cm}$, de modo que se maximiza el acceso a la luz. Estos sistemas operan con tiempos de residencia de entre 3 y 10 días, dependiendo de la disponibilidad de luz, la temperatura, la especie de microalga predominante y la naturaleza del agua residual.

Estos sistemas son los más extendidos en la depuración de aguas ya que ofrecen valores de velocidades de eliminación de nitrógeno, fósforo y materia orgánica bastante altos en relación a los costes de operación e implantación, y son muy sencillos de operar. Se estima que un raceway operando con un sistema mixto microalgas-bacterias consume entre $0-0,57 \mathrm{kWh} / \mathrm{kg}$ DBO frente a los sistemas de lodos activos que consumen entre 0,80 y 6,41 kWh/kg DBO eliminado (Rawat et al., 2011).

La productividad de biomasa algal cultivada en HRAP de gran escala $\left(>200 \mathrm{~m}^{3}\right)$ y cultivados tanto con fines comerciales como para depuración de aguas oscila en torno a los 12-40 $\mathrm{g} / \mathrm{m}^{2}$.día (Park et al., 2011) dependiendo de la especie cultivada, el clima y el modo de operación.

Los sistemas del tipo HRAP, al ser los más simples y económicos, son también los más usados en la depuración de aguas. En ellos se puede llegar a concentraciones de hasta 0,5 
$\mathrm{g} / \mathrm{L}$ tanto para cultivos puros como en cultivos mixtos de bacterias y microalgas (Chisti, 2013; Komolafe et al., 2014). La mejora en la productividad del crecimiento de biomasa y los rendimientos de eliminación de contaminantes en esta tecnología se basan principalmente en la instalación de mejoras y en la optimización de parámetros de operación en los HRAP.
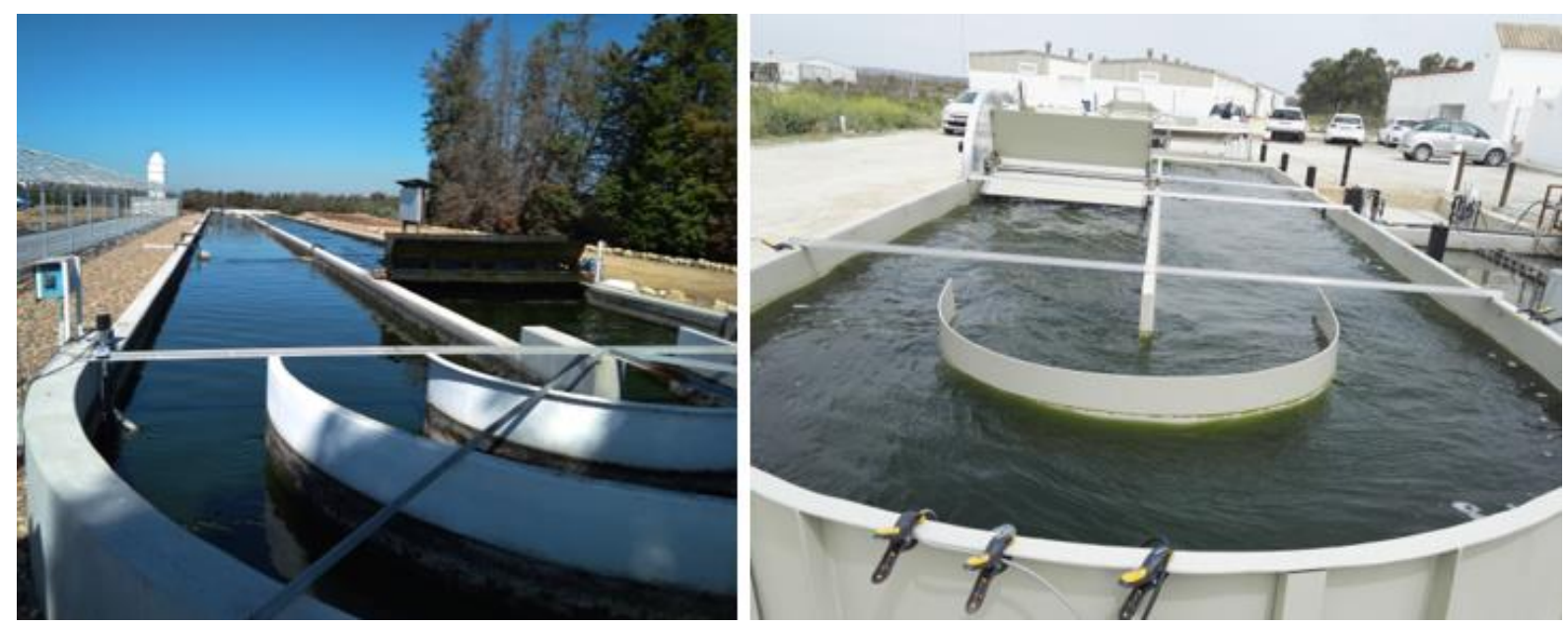

Figura 3. HRAP de $116 \mathrm{~m}^{3}$ ubicado en las instalaciones del CENTA (Centro de las Nuevas Tecnologías del Agua), Carrión de los Céspedes (Sevilla, España), y HRAP piloto de $6 \mathrm{~m}^{3}$ ubicado en las instalaciones de CUPIMAR (Cultivos Piscícolas Marinos) Puerto Real (Cádiz, España)

\section{Geometría}

Debido a que estos sistemas se han posicionado como los más adecuados para la producción de microalgas a gran escala, la optimización del diseño hacia mayores productividades aéreas se ha convertido en uno de los principales objetivos. Además de la distribución de la luz, las características hidrodinámicas son críticas para obtener altas productividades. En el diseño clásico o básico (Figura 4) aparecen zonas muertas y una distribución no uniforme de la velocidad. Las zonas muertas son zonas en las que el flujo está estancado y se puede producir el crecimiento indeseado de bacterias anaeróbicas que influyen de forma negativa en la eficiencia del cultivo (Shilton, 2005). 


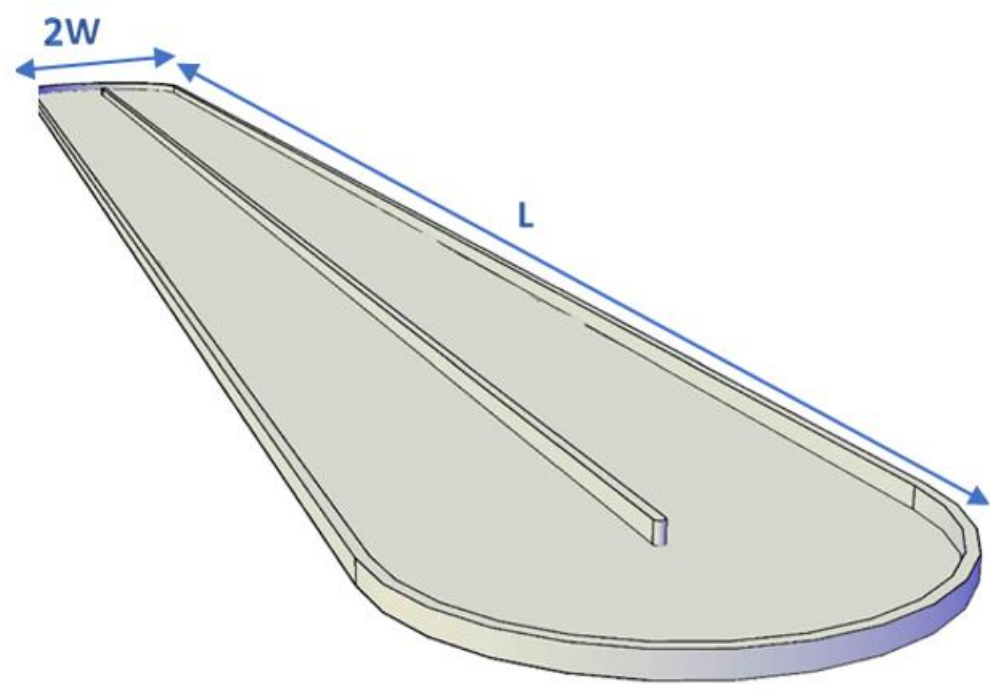

Figura 4. Geometría de un HRAP básico

En el diseño de un HRAP uno de los parámetros geométricos clave es la relación largo/ancho (L/W) del canal, que debe ser mayor de 10 para que exista una mezcla y distribución del medio adecuada (Weissman et al., 1988).

El intervalo de profundidad para el mejor aprovechamiento de luz está entre 10 y $15 \mathrm{~cm}$, de este modo se tienen productividades más altas al eliminar el auto-sombreado y la estratificación térmica (Grobbelaar, 2012). Sin embargo, el intervalo de profundidades operativos a gran escala suele estar entre 20 y $50 \mathrm{~cm}$ de profundidad (Brennan y Owende, 2010). De esta forma se reduce la influencia de las paredes y el suelo sobre la velocidad del flujo.

Los giros con geometría curva normalmente de $180^{\circ}$, minimizan las zonas muertas si bien persisten estas zonas debido a la reducción de la velocidad del fluido. Añadiendo bafles se homogeniza el flujo en las curvas y se elimina con éxito una alta proporción de las zonas muertas (Andersen, 2005; Grobbelaar, 2012).

A parte de la geometría, el material de los HRAP también es un parámetro que puede influir en la productividad. Éstos suelen estar construidos de hormigón o de tierra compactada 
recubierta por un material plástico, es importante que el material ofrezca la menor fricción posible para reducir el consumo energético.

\section{Impulsión y Mezcla}

Un cultivo homogéneo lleva asociada una productividad más alta, debido a un óptimo acceso a los nutrientes, una mejor distribución de la luz entre los microorganismos (menor fotoinhibición y autosombreado) y prácticamente, nula sedimentación de células en el fondo. Esta homogeneidad se consigue principalmente con un buen sistema de impulsión que origine un flujo turbulento a través de los canales. Un flujo laminar favorece la sedimentación de las células e incrementa la presencia de zonas muertas (Becker, 2008) además de favorecer las exposiciones extremas a la luz (mucha intensidad en la superficie y oscuridad en el fondo), que es lo que se pretende evitar.

Aunque una velocidad de flujo mayor lleva asociado mejor mezcla del cultivo, si ésta es demasiado alta, puede limitar el crecimiento debido al estrés provocado sobre las células, que pueden incluso llegar a romperse (Barbosa et al., 2004). Además, mayor velocidad supone un mayor consumo energético, siendo una función cúbica de la velocidad (Hadiyanto et al., 2013). Para cumplir con todos estos limitantes, la velocidad en los canales suele estar limitada a valores entre 10 y $40 \mathrm{~cm} / \mathrm{s}$.

La impulsión del medio a través de los canales viene dada generalmente por una rueda de paletas. Estos sistemas consisten en una serie de paletas que giran sobre un eje horizontal con la potencia aportada por un motor (del orden de $2 \mathrm{~W}$ por cada $\mathrm{m}^{3}$ de reactor (Borowitzka, 2005). Son sistemas mecánicamente simples y requieren poco mantenimiento además de que proporciona una mezcla suave que no daña las células.

\section{Monitorización}

Un fotobiorreactor del tipo HRAP es un equipo con bajos requerimientos en términos de mantenimiento. Los únicos equipos mecánicos que posee es el motor que impulsa la rueda de palas, y si fuese el caso (en el tratamiento de aguas residuales de bajo contenido en carbono orgánico), el mecanismo de aireación y/o difusión de $\mathrm{CO}_{2}$. 
Por otra parte, como la mayoría de los procesos extensivos de tratamiento de aguas residuales urbanas, presenta una elevada dependencia de las condiciones climáticas, en este caso, especialmente de la temperatura y la irradiancia solar. Conviene pues llevar un seguimiento periódico del fotobiorreactor, siendo los parámetros más característicos los incluidos en los siguientes apartados.

\subsection{Oxígeno disuelto}

Una fotosíntesis intensa durante el día puede incrementar el nivel de oxígeno disuelto hasta más del 300\% de saturación en las horas de picos de radiación (Ippoliti et al., 2016). Esto tiene un impacto negativo sobre la productividad, ya que, a altas concentraciones, por encima de $20 \mathrm{mg} / \mathrm{L}$, el oxígeno puede inhibir la fijación de carbono en las células de microalgas. En el caso de HRAP alimentados con medio rico en carbono orgánico, estos son, sistemas de cultivos mixtos bacterias-microalgas, no suele alcanzarse concentraciones inhibitorias de oxígeno debido a la actividad metabólica de las bacterias (Arbib et al., 2016).

\subsection{Temperatura}

La mayoría de los bioprocesos son muy sensibles a los cambios de temperatura, tanto diarios como estacionales. En un HRAP, aunque sea una variable no controlable conviene registrar la temperatura del cultivo y del ambiente para disponer de datos que expliquen cambios en el comportamiento del reactor, de este modo establecer patrones que nos indiquen los tiempos de respuesta del sistema ante cambios ambientales de temperatura.

\section{$5.3 \mathrm{pH}$}

A lo largo del día y conforme se aumenta la actividad fotosintética, el pH del medio de cultivo tiende a desplazarse hacia pH básicos (Figura 5). En monocultivos de microalgas sin control de $\mathrm{pH}$, se pueden alcanzar valores de 11 (Becker, 2008) aunque lo normal es que el valor no supere valores de 9 en cultivos mixtos microalgas-bacterias (Arbib et al., 2016). En los HRAP más sofisticados el $\mathrm{pH}$ puede ser controlado mediante la difusión de $\mathrm{CO}_{2}$, pero esto encarece el proceso (CAPEX y OPEX) y solo podría estar justificado si lo que se pretende es producir biomasa algal de valor. 


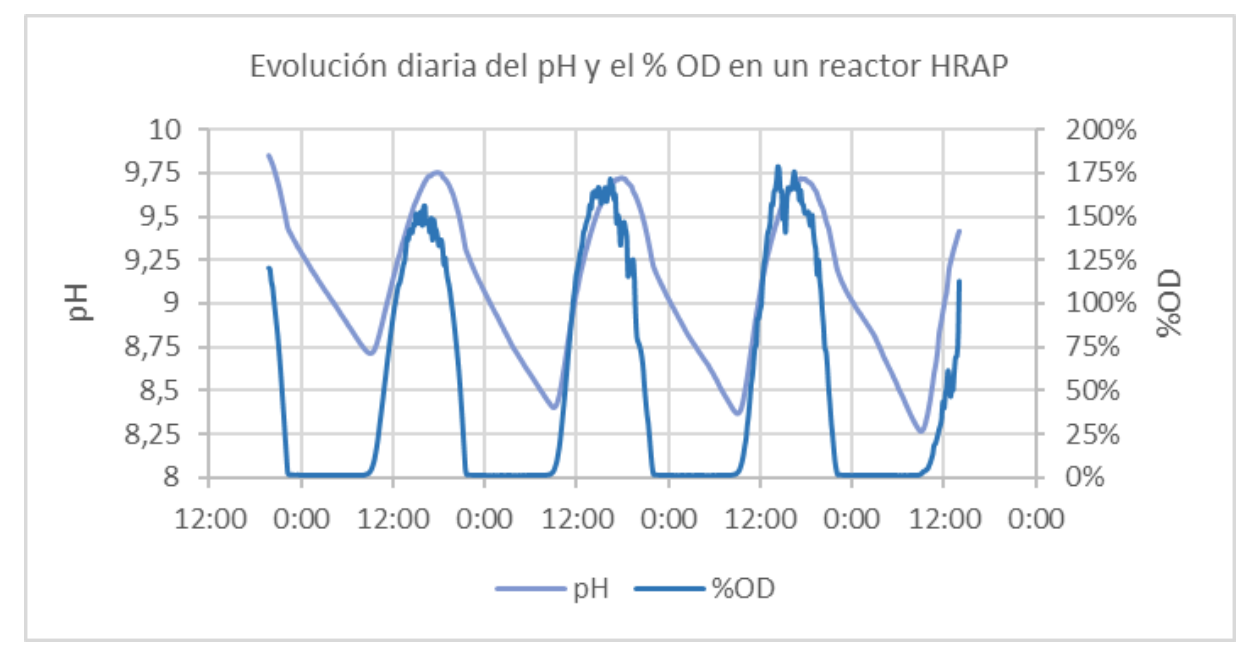

Figura 5. Variaciones diarias de oxígeno disuelto y $\mathrm{pH}$ en el reactor HRAP del CENTA, Carrión de los Céspedes (Sevilla, España), del 2 al 6 de julio de 2018

\subsection{Luz}

La disponibilidad de luz es el principal factor limitante en los cultivos de microalgas. Para medirla se debe cuantificar la radiación fotosintéticamente activa (PAR), aquella comprendida entre 400 y $700 \mathrm{~nm}$, que es la que la mayoría de organismos fotosintéticos puede utilizar para realizar la fotosíntesis. La densidad de flujo de fotones fotosintéticos (PPFD) se puede medir empleando sensores denominados Quantum pAE y los datos se pueden expresar en $\mu \mathrm{mol} / \mathrm{m}^{2} \cdot \mathrm{s}^{1}$ (Figura 6). Para que el crecimiento del cultivo sea adecuado, la irradiancia media que reciba la instalación no debería estar por debajo de los $100-150 \mu \mathrm{mol} / \mathrm{m}^{2} \cdot \mathrm{s}^{1}$, pues de lo contrario la demanda de espacio se vería incrementada considerablemente debido a la baja velocidad de crecimiento de las microalgas. 


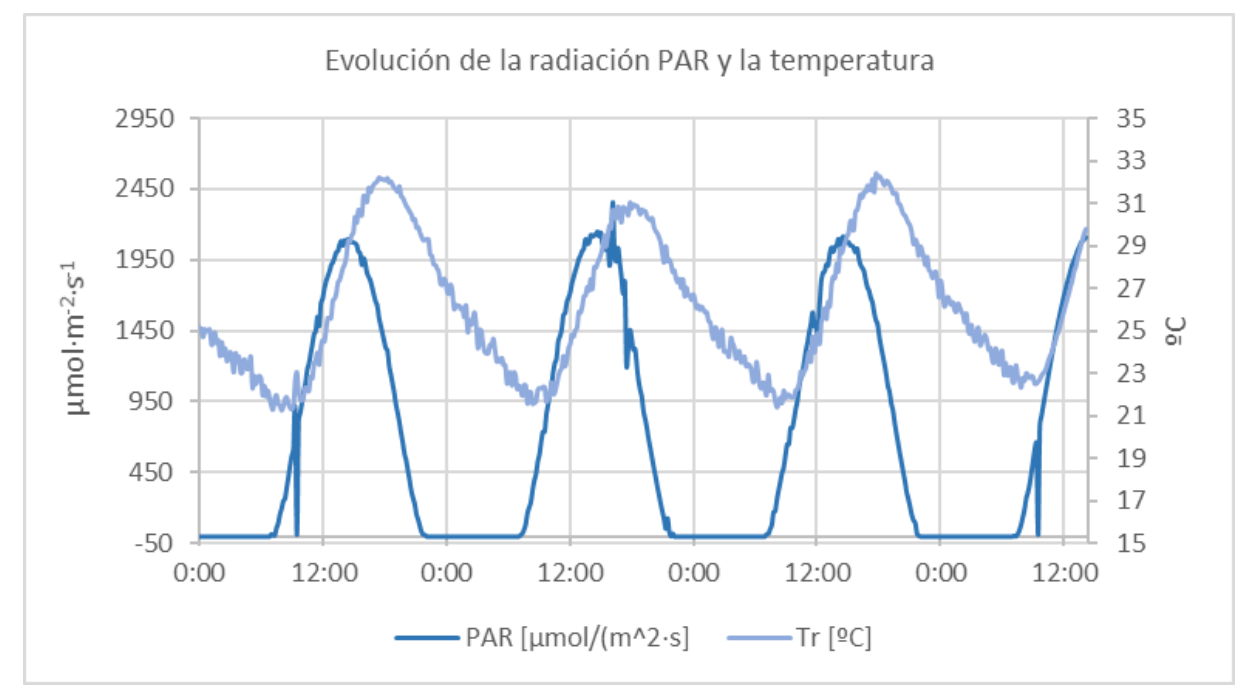

Figura 6. Evolución diaria de la temperatura y la radiación PAR del 2 al 6 de julio de 2018 en Carrión de los Céspedes, Sevilla

\subsection{Biomasa}

La concentración de biomasa en el medio debe ser controlada. Concentraciones bajas se traducen en menores tasas de eliminación de nutrientes. Por lo general, el tiempo de residencia para la eliminación de nutrientes es mucho más bajo que el de crecimiento de biomasa (mayor velocidad de consumo de $\mathrm{N}$ y $\mathrm{P}$ que de crecimiento), por lo que en sistemas sin recirculación de biomasa (mismo tiempo hidráulico de residencia que de retención celular), es la cinética de crecimiento de la biomasa la que rige el tiempo hidráulico de residencia de operación (Ruiz et al., 2014). Por lo general, en fotobiorreactores del tipo HRAP se pueden llegar a concentraciones de 0,6 g/L SS (Tredici, 2004), pero en cultivos en aguas residuales suele obtenerse valores de 0,2-0,4 g/L (Arbib et al., 2016; Mehrabadi et al., 2016), siendo la disponibilidad de luz un factor limitante, pues a mayor concentración de biomasa, mayor es el efecto de autosombreado (Ruiz et al., 2014). Se puede hacer seguimiento de este parámetro mediante análisis periódicos de sólidos en suspensión o mediante un turbidímetro calibrado. 


\subsection{Quantum yield o rendimiento cuántico.}

Es una sencilla medida de la actividad fotosintética de las microalgas en la que se puede conocer de forma rápida si hubo algún factor ambiental o estrés físico-químico que tuvo efectos inhibitorios sobre el aparato fotosintético.

\section{Cosechado}

La viabilidad de un sistema de depuración de aguas residuales basado en la biotecnología de microalgas depende no solo del desarrollo de un fotobiorreactor con un consumo energético y una demanda de espacio competitivos, sino también en la separación de la biomasa del agua residual ya tratada, pues éste es un proceso determinante que impacta directamente no sólo en la calidad del efluente sino en los costes de inversión y operación de todo el proceso.

Las concentraciones de microalgas que se encuentran comúnmente en reactores HRAP (< 0,5 g/L en Chisti (2013)), requieren un procesamiento de grandes volúmenes que pueden conducir a elevados costes de producción. Disminuir el caudal a procesar mediante una adecuada fase de pre-concentrado es vital para la viabilidad de estas tecnologías de depuración.

La selección de una tecnología adecuada de separación depende de muchos factores, incluyendo el tipo de célula, la densidad y el tamaño, junto con los requisitos de procesamiento posteriores y el uso que se va a realizar de la biomasa generada (Brennan y Owende, 2010; Singh y Patidar, 2018). Se han ideado muchas técnicas de separación durante las últimas cuatro décadas, sin embargo, en general se pueden dividir en tecnologías que se utilizan en la etapa de preconcentración o separación primaria, y en la etapa de deshidratación o separación secundaria. Durante una etapa de separación primaria, por lo general, se alcanzan concentraciones de microalgas del orden de $2-7 \%$ de SS, y esto se puede lograr mediante floculación (Vandamme et al., 2013), flotación (Laamanen et al., 2016) y/o sedimentación (Barros et al., 2015). Esta separación se continua con un segundo paso de deshidratación o espesamiento, que produce una torta de algas con un 15-25\% de SS. Para esta etapa se suelen emplear tecnologías basadas 
en la filtración o centrifugación, y es a menudo un proceso más intensivo energéticamente que los empleados en la separación primaria.

\subsection{Centrifugación}

La centrifugación de la biomasa de microalgas procedente de la depuración de aguas residuales, posiblemente no sea la mejor tecnología debido al consumo energético que supone. Estos valores dependen del tipo de centrífuga utilizada, la especie de microalgas y la fase de cosechado (una sola etapa o dos etapas), estando comprendido entre 0,7-8 kWh/m³ (Molina Grima et al., 2003; Milledge y Heaven, 2013).

\subsection{Filtración}

La macro-filtración se utiliza cuando en el cultivo hay especies de gran tamaño o coloniales (p.ej. Arthrospira o Anabaena). Los filtros banda pueden llegar a concentraciones del 20\% con un consumo energético de tan solo $0,5 \mathrm{kWh} / \mathrm{m}^{3}$ siempre y cuando la alimentación esté preconcentrada al 4\% (Vandamme et al., 2013). En general, aunque no hay un consenso generalizado, se podría afirmar que la mayoría de los autores consideran que la microfiltración tiene el tamaño de poro más apropiado para retener la mayoría de las especies más comunes de microalgas. Si bien, Molina Grima et al. (2003) afirman que la microfiltración podría ser incluso menos económica que la centrifugación para la recuperación de células de microalgas a gran escala. Bilad et al. (2012) demostraron que la recolección mediante filtración sumergida en combinación con centrifugación podría lograr una concentración de hasta el $22 \%$ y reducir las necesidades de energía por debajo de $1 \mathrm{kWh} / \mathrm{m}^{3}$.

\subsection{Flotación}

Coward et al. (2013) encontraron que la flotación tiene potencial como método de concentración primario, consiguiendo concentrar Chlorella sp. hasta 2,5\% con un consumo energético tan bajo como 0,015 kWh/m³. En Chiclana de la Frontera (Cádiz, España) se ha desarrollado el proyecto "AllGas" financiado por el 7ํㅡㄹ Programa Marco de la Unión Europea sobre de depuración de aguas residuales urbanas a escala demostrativa mediante un cultivo mixotrófico microalgas-bacterias. En este proyecto, el cosechado se realiza 
mediante flotación obteniendo concentraciones de hasta un $4 \%$ y con un consumo energético inferior a $0,04 \mathrm{kWh} / \mathrm{m}^{3}$ (Arbib et al., 2016).

\subsection{Sedimentación}

Este método presenta los menores costes de inversión, así como bajos requisitos de capacitación de los operadores. Para una microalga de forma esférica, por ejemplo, del género Chlorella, la velocidad de sedimentación calculada sería del orden de 0,1 $\mathrm{m} /$ día (Milledge y Heaven, 2013). En un estudio sobre 24 microalgas autótrofas con tamaños comprendidos entre los 10 y 1.000 um (Peperzak et al., 2003) se determinó que la velocidad de sedimentación variaba entre 0,4 y 2,2 m/día.

La recuperación celular usando sedimentación es generalmente baja, 60-65\% con una concentración de sólidos en el lodo de hasta 1,5\% (Uduman et al., 2010). La velocidad de sedimentación, la recuperación celular y la concentración de sólidos pueden mejorarse induciendo una floculación antes de la sedimentación.

\subsection{Coagulación - Floculación}

En la coagulación-floculación de microalgas se suele emplear coagulantes y floculantes catiónicos para neutralizar la carga superficial negativa de las células y facilitar la formación espontánea de agregados celulares o flóculos. Los coagulantes catiónicos incluyen sales de iones metálicos multivalentes tales como sulfato de aluminio y cloruro férrico (Gorin et al., 2015). Los polímeros catiónicos son otros floculantes eficaces y ampliamente utilizados (Mennaa et al., 2017), pero en general son mucho más caros que las sales metálicas, si bien su dosificación es más baja. El empleo de coagulantes y floculantes para el cosechado de microalgas es una técnica que, aunque eficaz como sistema de concentración primaria (se alcanzan capturas del orden del $90 \%$, con concentraciones de lodo comprendidas entre un 1 y un 3\%), los costes de operación se disparan debido al consumo de reactivos (OPEX). Mennaa et al. (2017) determinaron costes de cosechado de microalgas cultivadas en agua residual asociados solamente al consumo de reactivos del orden de $0,02-0,023 € / \mathrm{m}^{3}$ dependiendo del coagulante o floculante empleado. 
Los flóculos mixtos microalgas-bacterias pueden ayudar a las microalgas a sedimentar más rápido (Van Den Hende et al., 2011). Tanto las bacterias como las algas pueden producir sustancias poliméricas extracelulares (EPS) que favorecen la auto o biofloculación (Lee et al., 2013; Singh y Patidar, 2018).

Aplicado a la depuración de aguas residuales, el proceso de separación de la biomasa algal del agua ha de presentar una elevada eficacia de funcionamiento y estabilidad ante fluctuaciones de caudal. El proceso de separación ha de cumplir que sea simple de mantener y explotar, y no requiera personal cualificado. Para ello ha de contar con los mínimos equipos electromecánicos, de modo que también se minimicen los costes de mantenimiento y explotación. A la vista de los costes energéticos y de consumo de reactivos de las diferentes alternativas, parece ser que la opción de un proceso de biofloculación como consecuencia del cultivo mixotrófico bacterias-microalgas seguido de un proceso de sedimentación, como primera fase de separación podría ser una opción que hiciese el proceso viable. Para la deshidratación de los lodos generados, las opciones podrían abarcar desde una era de secado hasta una centrífuga tipo decanter en el caso de que la biomasa generada tuviese un valor lo suficientemente elevado como para justificar económicamente la inversión y los costes de explotación. Por lo general, si la pluviometría lo permite, se debería optar por las eras de secado por encima del uso de una centrífuga.

\section{Perspectiva de la depuración de aguas residuales en pequeñas poblaciones mediante tecnología de microalgas}

En la depuración de aguas residuales urbanas de pequeños municipios, la aplicación de tecnología de microalgas en consorcio con bacterias y utilizando reactores HRAPs, tiene una gran aplicabilidad futura debido a que es una tecnología sencilla, fácil de operar y que garantiza unos efluentes depurados tanto en materia orgánica como en carga de nutrientes, además es una tecnología fijadora de $\mathrm{CO}_{2}$. Esta tecnología tiene un bajo coste de operación lo que la hace compatible con la aplicación a pequeños núcleos de población. No obstante, aún no se trata de una solución viable debido fundamentalmente a la fase de cosechado, donde es preciso profundizar en materia de I+D+i para buscar una solución poco tecnificada y compatible con instalaciones de depuración extensivas. 


\section{AGRADECIMIENTOS}

Este trabajo ha sido financiado a través del Proyecto internacional de potenciación de la $1+D+i$ de excelencia para la depuración de las aguas en pequeñas aglomeraciones urbanas (IDIAQUA) financiado a través del programa de la Unión Europea Interreg VA España-Portugal (POCTEP) de cooperación transfronteriza. Asimismo, quisiéramos agradecer su colaboración a la Fundación Pública Andaluza Centro de las Nuevas Tecnologías del Agua (CENTA).

\section{REFERENCIAS}

Andersen, R.A. \& Robert, A. (2005). Algal culturing techniques. Elsevier/Academic Press. ISBN: 0120884267.

Arbib, Z., de Godos, I., Ruiz, J. \& Perales, J.A. (2017). Optimization of pilot high rate algal ponds for simultaneous nutrient removal and lipids production. Sci. Total Environ. 589, 66-72. https://doi.org/10.1016/j.scitotenv.2017.02.206.

Arbib, Z., Godos, I. de, Lara, E., Fernandez, M., Rogalla, F. \& Llamas, B. (2016). Wastewater as Biofuel: Nutrient and Energy Recovery with Algae Treatment. Proc. Water Environ. Fed. 2015, 1-16. https://doi.org/10.2175/193864715819558802.

Arbib, Z., Ruiz, J., Álvarez Díaz, P., Garrido Pérez, C., Barragan \& J., Perales, J.A. (2013). Long term outdoor operation of a tubular airlift pilot photobioreactor and a high rate algal pond as tertiary treatment of urban wastewater. Ecol. Eng. 52, 143-153. https://doi.org/10.1016/j.ecoleng.2012.12.089.

Barbosa, M.J., Hadiyanto \& Wijffels R.H. (2004). Overcoming Shear Stress of Microalgae Cultures in Sparged Photobioreactors. Biotechnol. Bioeng. 85, 78-85. https://doi.org/10.1002/bit.10862.

Barros, A.I., Gonçalves, A.L., Simões, M. \& Pires, J.C.M. (2015). Harvesting techniques applied to microalgae: A review. Renew. Sustain. Energy Rev. 41, 1489-1500. https://doi.org/10.1016/j.rser.2014.09.037.

Becker, E.W. (2008). Microalgae: biotechnology and microbiology. Cambridge University Press. ISBN: 052106113X.

Bilad, M.R., Discart, V., Vandamme, D., Foubert, I., Muylaert, K. \& Vankelecom, I.F.J. (2014). Coupled cultivation and pre-harvesting of microalgae in a membrane photobioreactor (MPBR). Bioresour. Technol. 155, 410-417. https://doi.org/10.1016/j.biortech.2013.05.026. 
Bilad, M.R., Vandamme, D., Foubert, I., Muylaert, K. \& Vankelecom, I.F.J. (2012). Harvesting microalgal biomass using submerged microfiltration membranes. $\begin{array}{lll}\text { Bioresour. } & \text { Technol. } & \text { 343-352. }\end{array}$ https://doi.org/10.1016/J.BIORTECH.2012.02.009.

Bode, H. \& Grunebaum, T. (2000). The cost of municipal sewage treatment - Structure, origin, minimization - Methods of fair cost comparison and allocation. Water Sci. Technol. 41, 289-298. https://doi.org/10.2166/wst.2000.0226.

Borowitzka, M.A. (2005). Culturing microalgae in outdoor ponds. In: Andersen, I.R.A.(Ed.), Algal Culturing Techniques. Elsevier, Academic Press, New York, pp. 205-218.

Brennan, L. \& Owende, P. (2010). Biofuels from microalgae-A review of technologies for production, processing, and extractions of biofuels and co-products. Renew. Sustain. Energy Rev. 14, 557-577. https://doi.org/10.1016/j.rser.2009.10.009.

Carey, R.O. \& Migliaccio, K.W. (2009). Contribution of Wastewater Treatment Plant Effluents to Nutrient Dynamics in Aquatic Systems: A Review. Environ. Manage. 44, 205-217. https://doi.org/10.1007/s00267-009-9309-5.

Chisti, Y. (2013). Raceways-based production of algal crude oil. Green 3, 195-216. https://doi.org/10.1515/green-2013-0018.

Christenson, L.B. \& Sims, R.C. (2012). Rotating algal biofilm reactor and spool harvester for wastewater treatment with biofuels by-products. Biotechnol. Bioeng. 109, 16741684. https://doi.org/10.1002/bit.24451.

Collado Lara, R. (1992). Depuración de aguas residuales en pequeñas comunidades. Colegio de Ingenieros de Caminos, Canales y Puertos, D. L. ISBN: 84-380-0050-9.

Coward, T., Lee \& J.G.M., Caldwell, G.S. (2013). Development of a foam flotation system for harvesting microalgae biomass. Algal Res. 2, 135-144. https://doi.org/10.1016/J.ALGAL.2012.12.001.

Craggs, R.J., Adey, W.H., Jessup, B.K. \& Oswald, W.J. (1996). A controlled stream mesocosm for tertiary treatment of sewage. Ecol. Eng. 6, 149-169. https://doi.org/10.1016/0925-8574(95)00056-9.

Craggs, R.J., Heubeck, S., Lundquist, T.J. \& Benemann, J.R. (2011). Algal biofuels from wastewater treatment high rate algal ponds. Water Sci. Technol. 63, 660-665. https://doi.org/10.2166/wst.2011.100. 
Díaz-Garduño, B., Pintado-Herrera, M.G., Biel-Maeso, M., Rueda-Márquez, J.J., LaraMartín, P.A., Perales, J.A., Manzano, M.A., Garrido-Pérez \& C., Martín-Díaz, M.L. (2017). Environmental risk assessment of effluents as a whole emerging contaminant: Efficiency of alternative tertiary treatments for wastewater depuration. Water Res. 119, 136-149. https://doi.org/10.1016/j.watres.2017.04.021.

Doucha, J. \& Lívanský, K. (1995). Novel outdoor thin-layer high density microalgal culture system: Productivity and operational parameters. Arch. Hydrobiol. Suppl. Algol. Stud. 76, 129-147.

Duarte-Santos, T., Mendoza-Martín, J.L., Acién Fernández, F.G., Molina, E., Vieira-Costa \& J.A., Heaven, S. (2016). Optimization of carbon dioxide supply in raceway reactors: Influence of carbon dioxide molar fraction and gas flow rate. Bioresour. Technol. 212, 72-81. https://doi.org/10.1016/j.biortech.2016.04.023.

Gorin, K. V., Sergeeva, Y.E., Butylin, V. V., Komova, A. V., Pojidaev, V.M., Badranova, G.U., Shapovalova, A.A., Konova, I.A. \& Gotovtsev, P.M. (2015). Methods coagulation/flocculation and flocculation with ballast agent for effective harvesting of microalgae. Bioresour. Technol. 193, 178-184. https://doi.org/10.1016/J.BIORTECH.2015.06.097.

Grobbelaar, J.U. (2012). Microalgae mass culture: The constraints of scaling-up. J. Appl. Phycol. 24, 315-318. https://doi.org/10.1007/s10811-011-9728-6.

Hadiyanto, H., Elmore, S., Van Gerven, T. \& Stankiewicz, A. (2013). Hydrodynamic evaluations in high rate algae pond (HRAP) design. Chem. Eng. J. 217, 231-239. https://doi.org/10.1016/j.cej.2012.12.015.

Hoh, D., Watson, S. \& Kan, E. (2016). Algal biofilm reactors for integrated wastewater treatment and biofuel production: A review. Chem. Eng. J. 287, 466-473. https://doi.org/10.1016/J.CEJ.2015.11.062.

Ippoliti, D., González, A., Martín, I., Sevilla, J.M.F., Pistocchi, R. \& Acién, F.G. (2016). Outdoor production of Tisochrysis lutea in pilot-scale tubular photobioreactors. J. Appl. Phycol. 28, 3159-3166. https://doi.org/10.1007/s10811-016-0856-x.

Janssen, M., Tramper, J., Mur, L.R. \& Wijffels, H. (2003). Enclosed Outdoor Photobioreactors: Light Regime, Photosynthetic Efficiency, Scale-Up, and Future Prospects. https://doi.org/https://doi.org/10.1002/bit.10468. 
Johnson, M.B. \& Wen, Z. (2010). Development of an attached microalgal growth system for biofuel production. Appl. Microbiol. Biotechnol. 85, 525-534. https://doi.org/10.1007/s00253-009-2133-2.

Kesaano, M. \& Sims, R.C. (2014). Algal biofilm based technology for wastewater treatment. Algal Res. 5, 231-240. https://doi.org/10.1016/J.ALGAL.2014.02.003.

Komolafe, O., Velasquez Orta, S.B., Monje-Ramirez, I., Noguez, I.Y., Harvey, A.P. \& Orta Ledesma, M.T. (2014). Biodiesel production from indigenous microalgae grown in wastewater. Bioresour. Technol. 154, 297-304. https://doi.org/10.1016/j.biortech.2013.12.048.

Laamanen, C.A., Ross, G.M. \& Scott, J.A. (2016). Flotation harvesting of microalgae. $\begin{array}{lllll}\text { Renew. } & \text { Sustain. } & \text { 75-86. }\end{array}$ https://doi.org/10.1016/J.RSER.2015.12.293.

Lee, J., Cho, D.H., Ramanan, R., Kim, B.H., Oh, H.M. \& Kim, H.S. (2013). Microalgaeassociated bacteria play a key role in the flocculation of Chlorella vulgaris. Bioresour. Technol. 131, 195-201. https://doi.org/10.1016/j.biortech.2012.11.130.

Lee, Seung-Hoon, Oh, H.-M., Jo, B.-H., Lee, S.-A., Shin, S.-Y., Kim, H.-S., Lee, Sang-Hyup \& Ahn, C.-Y. (2014). Higher Biomass Productivity of Microalgae in an Attached Growth System, Using Wastewater. J. Microbiol. Biotechnol 24, 1566-1573. https://doi.org/10.4014/jmb.1406.06057.

Mehrabadi, A., Farid, M.M. \& Craggs, R. (2016). Variation of biomass energy yield in wastewater treatment high rate algal ponds. Algal Res. 15, 143-151. https://doi.org/10.1016/J.ALGAL.2016.02.016.

Mennaa, F.Z., Arbib, Z. \& Perales, J.A. (2017). Urban wastewater photobiotreatment with microalgae in a continuously operated photobioreactor: growth, nutrient removal kinetics and biomass coagulation-flocculation. Environ. Technol. 1-14. https://doi.org/10.1080/09593330.2017.1393011.

Milledge, J.J. \& Heaven, S. (2013). A review of the harvesting of micro-algae for biofuel production. Rev. Environ. Sci. Biotechnol. 12, 165-178. https://doi.org/10.1007/s11157-012-9301-z.

Molina Grima, E., Belarbi, E.-H., Acién Fernández, F.., Robles Medina, A. \& Chisti, Y., (2003). Recovery of microalgal biomass and metabolites: process options and economics. Biotechnol. Adv. 20, 491-515. https://doi.org/10.1016/S07349750(02)00050-2. 
Morales-Amaral, M. del M., Gómez-Serrano, C., Acién, F.G., Fernández-Sevilla \& J.M., Molina-Grima, E. (2015). Outdoor production of Scenedesmus sp. in thin-layer and raceway reactors using centrate from anaerobic digestion as the sole nutrient source.

Algal Res. 12, 99-108. https://doi.org/10.1016/J.ALGAL.2015.08.020.

Muñoz, R. \& Guieysse, B. (2006). Algal-bacterial processes for the treatment of hazardous contaminants: A review. Water Res. https://doi.org/10.1016/j.watres.2006.06.011.

Norsker, N.H., Barbosa, M.J., Vermuë, M.H. \& Wijffels, R.H. (2011). Microalgal production - A close look at the economics. Biotechnol. Adv. 29, 24-27. https://doi.org/10.1016/J.BIOTECHADV.2010.08.005.

Oswald, W.J. \& Golueke, C.G. (1960). Biological Transformation of Solar Energy. Adv. Appl. Microbiol. 2, 223-262. https://doi.org/10.1016/S0065-2164(08)70127-8.

Oswald, W.J., Gotaas, H.B., Golueke, C.G., Kellen, W.R. \& Gloyna, E.F. (1957). Algae in Waste Treatment. Sewage Ind. Waste. 29, 437-457. https://doi.org/10.2307/25033322.

Ozkan, A., Kinney, K., Katz, L. \& Berberoglu, H. (2012). Reduction of water and energy requirement of algae cultivation using an algae biofilm photobioreactor. Bioresour. Technol. 114, 542-548. https://doi.org/10.1016/J.BIORTECH.2012.03.055.

Park, J.B.K. \& Craggs, R.J. (2011). Algal production in wastewater treatment high rate algal ponds for potential biofuel use. Water Sci. Technol. 63, 2403-2410. https://doi.org/10.2166/wst.2011.200.

Peperzak, L., Colijn, F., Koeman, R., Gieskes, W.W.C. \& Joordens, J.C.A. (2003). Phytoplankton sinking rates in the Rhine region of freshwater influence. J. Plankton Res. 25, 365-383. https://doi.org/10.1093/plankt/25.4.365.

Posadas, E., García-Encina, P.A., Soltau, A., Domínguez, A., Díaz, I. \& Muñoz, R. (2013). Carbon and nutrient removal from centrates and domestic wastewater using algalbacterial biofilm bioreactors. Bioresour. Technol. 139, 50-58. https://doi.org/10.1016/j.biortech.2013.04.008.

Posadas, E., Morales, M. del M., Gomez, C., Acién, F.G. \& Muñoz, R. (2015). Influence of $\mathrm{pH}$ and $\mathrm{CO} 2$ source on the performance of microalgae-based secondary domestic wastewater treatment in outdoors pilot raceways. Chem. Eng. J. 265, 239-248. https://doi.org/10.1016/j.cej.2014.12.059.

Rawat, I., Ranjith Kumar, R., Mutanda, T. \& Bux, F. (2011). Dual role of microalgae: Phycoremediation of domestic wastewater and biomass production for sustainable 
biofuels production. Appl. Energy 88, 3411-3424. https://doi.org/10.1016/j.apenergy.2010.11.025.

Ruiz, J., Arbib, Z., Álvarez-Díaz, P.D., Garrido-Pérez, C., Barragán J. \& Perales, J.A. (2014). Influence of light presence and biomass concentration on nutrient kinetic removal from urban wastewater by Scenedesmus obliquus. J. Biotechnol. 178, 32-37. https://doi.org/10.1016/j.jbiotec.2014.03.001.

Salas, J., Pidre, J. \& Cuenca, I. (2013). Manual de tecnologías no convencionales para la depuración de aguas residuales, Journal of Chemical Information and Modeling. Centro de las Nuevas Tecnologías del Agua (CENTA). https://doi.org/10.1017/CBO9781107415324.004.

Shilton, A.N. (2005). Pond treatment technology. IWA Pub. https://doi.org/10.2166/9781780402499.

Singh, G. \& Patidar, S.K. (2018). Microalgae harvesting techniques: A review. Journal of Environmental Management. 217, 499-508.

Tchobanoglous, G., Burton, F.L. \& Metcalf \& Eddy. (1995). Ingeniería de aguas residuales: tratamiento, vertido y reutilización. McGraw-Hill, Interamericana de España.

Tredici, M.R. (2004). Mass production of microalgae: photobioreactors, in: Handbook of Microalgal Culture: Biotechnology and Applied Phycology. Blackwell Publishing Ltd.: Oxford, UK, pp. 178-214. https://doi.org/10.1002/9780470995280.ch9.

Uduman, N., Qi, Y., Danquah, M.K., Forde, G.M. \& Hoadley, A. (2010). Dewatering of microalgal cultures: A major bottleneck to algae-based fuels. J. Renew. Sustain. Energy 2, 012701. https://doi.org/10.1063/1.3294480.

Van Den Hende, S., Vervaeren, H., Desmet, S. \& Boon, N. (2011). Bioflocculation of microalgae and bacteria combined with flue gas to improve sewage treatment. $\mathrm{N}$. Biotechnol. 29, 23-31. https://doi.org/10.1016/J.NBT.2011.04.009.

Van Drecht, G., Bouwman, A.F., Harrison, J. \& Knoop, J.M. (2009). Global nitrogen and phosphate in urban wastewater for the period 1970 to 2050. Global Biogeochem. Cycles 23, 1-19. https://doi.org/10.1029/2009GB003458.

Vandamme, D., Foubert, I. \& Muylaert, K. (2013). Flocculation as a low-cost method for harvesting microalgae for bulk biomass production. Trends Biotechnol. 31, 233239. https://doi.org/10.1016/J.TIBTECH.2012.12.005. 
Villar-Navarro, E., Baena-Nogueras, R.M., Paniw, M., Perales, J.A. \& Lara-Martín, P.A. (2018). Removal of pharmaceuticals in urban wastewater: High rate algae pond (HRAP) based technologies as an alternative to activated sludge based processes. Water Res. 139, 19-29. https://doi.org/10.1016/J.WATRES.2018.03.072.

Wang, L., Li, Y., Chen, P., Min, M., Chen, Y., Zhu, J. \& Ruan, R.R. (2010). Anaerobic digested dairy manure as a nutrient supplement for cultivation of oil-rich green microalgae Chlorella sp. Bioresour. Technol. 101, 2623-2628. https://doi.org/10.1016/j.biortech.2009.10.062.

Wang, Y., Liu, J., Kang, D., Wu, C. \& Wu, Y. (2017). Removal of pharmaceuticals and personal care products from wastewater using algae-based technologies: a review. Rev. Environ. Sci. Biotechnol. 16, 1-19. https://doi.org/10.1007/s11157-017-9446$x$.

Weissman, J.C., Goebel, R.P. \& Benemann, J.R. (1988). Photobioreactor design: Mixing, carbon utilization, and oxygen accumulation. Biotechnol. Bioeng. 31, 336-344. https://doi.org/10.1002/bit.260310409.

Wilde, E.W. \& Benemann, J.R. (1993). Bioremoval of heavy metals by the use of microalgae. Biotechnol. Adv. 11, 781-812. https://doi.org/10.1016/0734-9750(93)90003-6.

Yu, R.Q. \& Wang, W.X. (2004). Biokinetics of cadmium, selenium, and zinc in freshwater alga Scenedesmus obliquus under different phosphorus and nitrogen conditions and metal transfer to Daphnia magna. Environ. Pollut. 129, 443-456. https://doi.org/10.1016/j.envpol.2003.11.013. 